\title{
Programa Bolsa Família: impacto das transferências sobre os gastos com alimentos em famílias rurais
}

\author{
Gisléia Benini Duarte ${ }^{1}$ \\ Breno Sampaio ${ }^{2}$ \\ Yony Sampaio ${ }^{3}$
}

Resumo: Programas de transferência condicionada de renda são políticas sociais correntemente empregadas para combater e reduzir a pobreza em diversos países. No curto prazo, esses programas visam aliviar os problemas decorrentes da situação de pobreza, sendo que, no longo prazo, o objetivo é investir no capital humano, quebrando o ciclo intergeracional da pobreza. Estudos têm sido realizados para avaliar os impactos desses programas sobre variáveis como freqüência escolar, trabalho infantil, gastos com alimentação, entre outros. Este trabalho avalia o impacto da transferência de renda do Programa Bolsa Família sobre os gastos com alimentos de famílias rurais. As estimações foram feitas com base no método de Propensity Score Matching (PSM), que corrige para o viés de seleção amostral. Os resultados mostram que o valor médio das despesas anuais para as famílias beneficiárias supera em $R$ \$ 246 os gastos totais das famílias não-participantes. Considerando que a média anual recebida por essas famílias é de $\mathrm{R} \$ 278$, pode-se inferir que $88 \%$ desse valor é utilizado para consumo de alimento. Portanto, o programa de transferência condicionada Bolsa Família exerce um impacto positivo sobre o consumo de alimentos dessas famílias selecionadas.

Palavras-chaves: Bolsa Família, transferência de renda, consumo alimentar, famílias rurais.

${ }^{1}$ Doutora em Economia, Departamento de Economia/Pimes, UFPE. E-mail: gisleiaduarte@gmail.com

${ }^{2}$ Doutorando em Economia, Departamento de Economia, University of Illinois. E-mail: brenosampaio@hotmail.com

${ }^{3}$ Professor Titular de Economia, Departamento de Economia/Pimes, UFPE. PhD. em Economia, Universidade da Califórnia, 1973. E-mail: sampyony@yahoo.com.br 


\begin{abstract}
Conditional income transfer programs are social policies currently adopted to reduce poverty in several countries. These conditional transfer schemes have a goal to alleviate some of the consequences of poverty in the short run and increase human capital in the long run changing the intergenerational poverty cycle. Several papers evaluate the impact of income transfer on school attendance, child work and food expenses, among others. This paper analyzes the impact of the Bolsa Familia Program on food expenses of rural families. The Propensity Score Method was used to correct sample selection bias. Results show that annual food expenses increased 246 reais in relation to non participant families. Considering that average annual transfer to these families is 278 reais, it can be concluded that $88 \%$ of the transfer is expend in food products. Thus, Bolsa Familia brings a positive impact on food consumption of the benefited families.
\end{abstract}

Key-words: Bolsa Família, conditional income transfer, food expenses, rural families.

Classificação JEL: I38, Q18.

\title{
1. Introdução
}

Programas de transferência de renda iniciam-se, no Brasil, em 1995, seguindo o modelo proposto pelo senador Eduardo Suplicy em 1991, denominado Programa de Garantia de Renda Mínima. Segundo Bourguignon, Ferreira e Leite (2003), programas de proteção social que garantem à população uma renda mínima apresentam, entre outras vantagens, mecanismos que não afetam os aspectos alocacionistas do mercado.

Em 2000, os gastos federais com esse programa representavam pouco mais de 0,25\% dos dispêndios com transferências sociais e 18\% dos gastos com assistência social. Portanto, até aquele período, o perfil das políticas de combate à pobreza não havia sido alterado. Considera-se que, até 2001, os programas de garantia de renda mínima eram basicamente de cunho municipal, condicionando a transferência à freqüência a escola e postos de saúde.

O Programa Bolsa Escola, quando implantado, tinha como objetivo elevar a participação escolar das crianças pertencentes aos domicílios com renda mensal inferior a $\mathrm{R} \$ 100$, por meio do repasse de um valor mensal por criança em idade escolar. Pretendia-se investir em capital humano, quebrando o ciclo intergeracional da pobreza. O Bolsa Alimentação, por sua vez, buscava estimular gestantes, nutrizes e crianças menores a participarem de atividades ligadas à área da saúde, englobando consultas pré-natal e pós-parto bem como programas educativos de aleitamento materno e alimentação saudável, reforçadores da melhoria do capital humano. Para tal, ofereciam uma transferência condicionada à família e à criança participante. 
No início de 2004, criou-se o Programa Bolsa Família, o qual incorporou o Bolsa Escola e outros como o Bolsa Alimentação, centralizando a administração de todos os dispositivos de transferência de renda direta do governo federal. Em 2005, o Bolsa Família foi expandido, atingindo cerca de 7 milhões de famílias. Objetivou-se atender a dois grupos: o de famílias com renda per capita mensal inferior a R\$50 sem nenhuma condicionalidade e o de famílias com uma renda per capita mensal entre $\mathrm{R} \$ 50$ e $\mathrm{R} \$ 100$ com crianças de até cinco anos. Até agosto de 2006, os domicílios selecionados pelo programa recebiam de $\mathrm{R} \$ 15$ a $\mathrm{R} \$ 95$. Para as famílias com renda inferior a $R \$ 50$, o valor mensal recebido era de $R$ \$ 50 mais $\mathrm{R} \$ 15$ por criança, sendo que para as famílias com renda per capita inferior a $R \$$ $100^{4}$, tal benefício era concedido para, no máximo, três crianças.

O Bolsa Família, assim como os programas anteriores de transferência de renda, partiu da constatação de que os níveis de pobreza da população brasileira não apresentaram nenhuma redução significativa nas últimas décadas do século passado (XX). Entre os motivos destacam-se o alto nível de desigualdade de renda e a estagnação do PIB, principalmente no período de 1980 a 1993. Somente com a implantação do Plano Real, em 1993, é que a economia brasileira ganhou mais estabilidade, resultando em um crescimento significativo de $11,61 \%$ no PIB nacional e afetando positivamente os níveis de pobreza. Daquele ano até 1999, o percentual de pobres no País reduziu-se em sete pontos percentuais. No entanto, $29 \%$ da população ainda vivia com renda per capita abaixo da linha de pobreza. Quando comparado a países com nível semelhante de desenvolvimento, o grau de pobreza no Brasil é bastante elevado (CAMARGO, 2003).

Nesse contexto, emerge a visão de que a política pública teria um papel crucial a cumprir: prevenir, enfrentar e reduzir a situação de pobreza das famílias. Adquirem importância as políticas de perfil redistributivo, que criam redes de proteção social para populações mais pobres. No entanto, face ao fracasso de políticas de distribuição direta de bens na redução da pobreza no curto prazo, ocorre a substituição dos mesmos por programas de transferência direta de renda.

Essa mudança de foco dos programas de transferência se deu também em decorrência do debate sobre a perspectiva da proteção social, o qual discute a importância de ações que constituem investimento em capital humano ao mesmo tempo em que fomentam o acesso a serviços básicos e buscam quebrar o ciclo da pobreza entre gerações. Os beneficiários desse sistema de proteção social são pessoas que vivem em pobreza estrutural, encontram-se abaixo da linha de pobreza e pertencem a grupos com necessidades especiais (VILLATORO, 2005).

Os programas de transferência condicionada enquadram-se no conceito de proteção social, visto que almejam elevar o investimento em capital humano. Esses programas têm como pressuposto o fato de que a reprodução da pobreza deve-se à

\footnotetext{
${ }^{4}$ Esses valores foram alterados em agosto de 2006, com o beneficio mínimo por família passando de $\mathrm{R} \$ 15$ para $\mathrm{R} \$ 18$, e o máximo, de $\mathrm{R} \$ 54$. O valor do beneficio básico subiu de $R \$ 50$ para $R \$ 58$, enquanto o teto máximo saltou de $R$ \$ 95 para $R \$ 112$.
} 
falta de investimento nas capacidades dos indivíduos; por isso buscam, por meio do condicionamento da transferência, gerar incentivos para esses investimentos (Villatoro, 2005). A condição de entrada no sistema se restringe, quase sempre, à remuneração, implicando na transparência da declaração da renda real e do patrimônio de cada requerente. O critério renda é largamente criticado na literatura pelos desvios que pode causar (MULLER; BIBI, 2005; MELO, 2008).

Esses programas de transferência de renda apresentam três principais estratégias: prevenção, enfrentamento e suavização da pobreza. Segundo Ravallion (2001), as estratégias de enfrentamento são orientadas para atenuar o impacto do risco ao qual as pessoas ou comunidades pobres estão expostas, uma vez que a situação de pobreza já existe. As estratégias de suavização e prevenção, por sua vez, são adotadas para diminuir os riscos futuros.

A importância desses programas de transferência no Brasil e o amadurecimento da literatura sobre condicionalidades estimularam o acompanhamento e a avaliação dos mesmos.

Diversos estudos analisaram o programa de transferência de renda Bolsa Escola. Cardoso e Souza (2004) avaliam o impacto do programa condicional de transferência de renda sobre a taxa de matrícula e a freqüência escolar das crianças pertencentes às famílias beneficiadas. Ao aplicar o método Propensity Score Matching (PSM), obtém-se como resultado que a participação das famílias no Bolsa Escola apresenta efeito positivo sobre a taxa de matrícula e a freqüência escolar. Bourguignom et. al (2003), usando modelo de micro-simulação ex ante, encontram um forte efeito da condicionalidade (freqüência escolar) sobre o comparecimento à escola, mas não encontram maior impacto das transferências sobre a pobreza e a distribuição de renda.

Outros estudos também apontam resultados positivos para o Programa Bolsa Escola. Segundo Ferro e Kassouf (2004), que avaliam o impacto do programa sobre o trabalho infantil, as crianças que participam do programa trabalham, em média, 3,4 horas a menos que as que não participam. O Bolsa Escola teria, portanto, contribuído para a redução da jornada de trabalho infantil. Villatoro (2005) identifica que o programa contribui positivamente para a acumulação de capital humano, para maior freqüência escolar e para a melhoria da relação idade/ano escolar.

Para o Bolsa Alimentação, também há avaliações (MINISTÉRIO DA SAÚDE, 2004; 2005) que constatam impacto positivo das transferências sobre o gasto com alimentação e sobre a diversificação da dieta, mas não foi constatado impacto sobre a utilização dos serviços de saúde, um dos objetivos centrais do programa.

Dada a importância já atribuída aos programas de transferência de renda, o presente estudo pretende analisar o impacto dessa transferência sobre os gastos com alimentos por parte das famílias beneficiadas. 
Segundo Attanasio et. al (2004), o efeito positivo das transferências do programa sobre o consumo pode não ocorrer de forma imediata. Isso porque a renda disponibilizada pelo programa não necessariamente aumenta na mesma proporção que o montante da renda familiar, dadas as condicionalidades do programa que podem reduzir outras fontes de renda, como a proveniente do trabalho infantil. Entretanto, o resultado que se espera é que as transferências influenciem de forma positiva o consumo e, conseqüentemente, o bem-estar das famílias beneficiadas.

Esse impacto pode ser maior que o de uma transferência de renda não-condicionada se a família entender que a transferência deve ser utilizada predominantemente em favor das crianças ou da melhoria da saúde. Isso pode ser uma decorrência de quem decide o gasto da renda da transferência - se a mulher ou o homem. Dessa forma, a elasticidade-renda por alimento pode ser maior para a renda transferida em relação à renda do trabalho. No caso do Bolsa Alimentação, a condicionalidade é explícita, e se constata aumento do gasto com alimentação para famílias com mesma renda, indicando que as elasticidades são distintas. Mas com o Bolsa Família, essa condicionalidade é, em partes, perdida. Uma hipótese possível é a de que mesmo sem condicionalidade esse comportamento se deva ao tomador de decisão do gasto desta renda de transferência. De modo geral, é atribuído papel crucial à mãe como tomadora de decisão quanto aos recursos de transferência do Bolsa Família.

A seção seguinte apresenta uma descrição da metodologia utilizada para análise bem como os dados e variáveis selecionados. Na terceira seção, são apresentados os resultados obtidos com o modelo propensity score. Por fim, a quarta seção traz as conclusões do trabalho.

\section{Metodologia}

Os estudos existentes relacionados aos impactos de políticas públicas sobre variáveis de interesse apontam como principal dificuldade a falta de informação a respeito dos indivíduos em situações diferentes, ou seja, dos indivíduos beneficiados e não beneficiados pela política.

Toma-se como exemplo a família $i$, com variável de interesse $Y$ (crescimento do consumo de alimentos). Têm-se duas situações: $D=1$ para os indivíduos que participarem da política e $D=0$, caso contrário. O resultado observado para a variável de interesse pode ser representado por:

$$
Y_{i}=D . Y_{1 i}+(1-D) . Y_{0}
$$


O impacto médio da política para o indivíduo $i$ e o impacto médio da política sobre a família beneficiada podem ser representados por:

$$
\Delta i=Y_{\downarrow} 1 i-Y_{\downarrow} 01 \text { e } \Delta i=E(\Delta i \mid D=1)=E\left(Y_{\downarrow} 1 i-Y_{\downarrow} 01 \mid D=1\right)
$$

em que $E(\cdot \mid D=1)$ refere-se ao valor esperado condicional à participação no programa social.

Como não é possível observar as famílias nas duas situações, utiliza-se nas avaliações um grupo das que não receberam o beneficio, o grupo de controle, obtendo-se uma medida aproximada do impacto do benefício sobre a variável considerada:

$$
\begin{aligned}
& E\left(Y_{1 i} \mid D=1\right)-E\left(Y_{0 i} \mid D=0\right)= \\
& =E\left(Y_{1 i} \mid D=1\right)-E\left(Y_{0 i} \mid D=1\right)+E\left(Y_{0 i} \mid D=1\right)-E\left(Y_{0 i} \mid D=0\right)= \\
& =\Delta+E\left(Y_{0 i} \mid D=1\right)-E\left(Y_{0 i} \mid D=0\right)
\end{aligned}
$$

A equação (1) expressa o viés ou a medida do erro que se incorre ao diferenciar as famílias apenas pelo fato destas participarem ou não da política. Uma simples comparação entre os grupos pode refletir diferenças pré-programa, e o efeito do programa pode ser decorrente de variáveis como idade e escolaridade dos indivíduos (ATTANASIO et al, 2004). Para a resolução do problema de viés de seleção, Rosenbaum e Rubin (1983) desenvolveram, a partir do matching ou pareamento das características observáveis dos indivíduos do grupo amostral, o método conhecido como Propensity Score Matching.

\subsection{Pareamento das Características Observáveis}

Neste trabalho, será utilizada a metodologia do matching ou pareamento das características observáveis. O matching é freqüentemente empregado nos estudos que avaliam o impacto de políticas sobre variáveis de interesse.

Esse método tem por finalidade encontrar um grupo de comparação ao grupo de tratamento a partir de uma amostra de indivíduos que não participam do programa ou política (grupo de controle). Tomam-se como base as características observáveis do grupo de tratamento, comparando-o com o grupo de controle, composto por indivíduos com características semelhantes às do grupo de tratamento.

Segundo Heckman et. al. (1997), o grupo de tratamento é representado por uma variável dummy $D$, que assume valor 1 caso o indivíduo tenha recebido o benefício e 0 caso contrário. A variável $Y_{1 i}$ denota a variável de interesse para o indivíduo $i$, que pertence ao grupo de tratamento (recebe o benefício) e $Y_{0 i}$ é o valor da mesma variável, caso o indivíduo não receba o benefício. Portanto, o 
impacto médio do programa sobre os beneficiados é dado pelo efeito médio do tratamento no (grupo) tratado (ATT):

$$
\begin{aligned}
& \Delta i=E\left(Y_{1 i}-Y_{0 i} \mid D_{i}=1\right) \\
& E\left(Y_{1 i}-Y_{0 i} \mid D_{i}=1\right)=E\left(Y_{1 i} \mid D_{i}=1\right)-E\left(Y_{0 i} \mid D_{i}=1\right)
\end{aligned}
$$

Considerando a equação (3), isso seria verdade se os grupos de controle e de tratamento fossem observados em duas situações no tempo. Como isso não é possível, admitem-se neste estudo algumas hipóteses sobre (3), como substituir o resultado da variável de interesse dos indivíduos beneficiados se o mesmo não tivesse sido beneficiado $E\left(Y_{0 i} \mid D_{i}=0\right)$. Porém, como é conhecido, os indivíduos beneficiados pela política de transferência social não foram escolhidos aleatoriamente e, portanto, é provável a existência de um viés. Logo $E\left(Y_{0 i} \mid D_{i}=1\right) \neq E\left(Y_{0 i} \mid D_{i}=0\right)$.

Para resolver o problema de viés, é necessário considerar as características observáveis $\left(X_{i}\right)$ que afetam a variável de interesse $(Y)$. Uma vez que os indivíduos com características observáveis idênticas possuem a mesma probabilidade de receber o benefício, o valor da variável de interesse $(Y)$ passa a ser independente do estado (se o indivíduo se encontra no grupo dos beneficiados ou controle). Formalmente, nessa situação tem-se $\left(Y_{1 i}, Y_{0 i} \perp D\right)$, e assim pode-se reescrever a equação 3 como:

$$
E\left(Y_{0 i} \mid X_{i}, D=1\right)=E\left(Y_{0 i} \mid X_{i}, D=0\right)=0
$$

Dessa forma, o efeito do programa sobre as famílias (equação 1) pode ser reescrita como: $\Delta=E\left(Y_{1 i} \mid X_{i}, D=1\right)-E\left(Y_{0 i} \mid X_{i}, D=0\right)$, tão logo o impacto médio do programa nas famílias seja obtido a partir da média ponderada dos resultados para os diversos grupos. Para isso, toma-se o valor esperado da equação 4, para o caso em que $D=1$, obtendo-se a expressão:

$$
\Delta=E\left[\left\{E\left(Y_{1 i} \mid X_{i}, D=1\right)-E\left(Y_{0 i} \mid X_{i}, D=0\right)\right] \mid D=1\right\}=E\{\Delta \mid D=1\}
$$

O impacto do programa sobre a variável de interesse $(Y)$ se dá por meio da soma ponderada das diferenças entre o valor médio dessa variável para beneficiados e não-beneficiados. Essa comparação é possível apenas para indivíduos com características semelhantes.

A dificuldade de aplicação desse método reside no fato de que, quanto mais características são utilizadas como base, maior será o número de observações necessário no grupo de tratamento para que possa ser feito o pareamento com o grupo de controle. Para eliminar esse problema, em vez de se utilizar as variáveis que expressam as características observáveis $\left(X_{i}\right)$, passou-se a considerar as probabilidades associadas a essas variáveis $P\left(X_{i}\right)$. Essa alteração foi realizada por Rosembaum e Rubin (1983) e o método ficou conhecido como Propensity Score Matching. 
Programa Bolsa Família: impacto das transferências

sobre os gastos com alimentos em famílias rurais

\subsection{Propensity Score Matching (PSM)}

O método de avaliação do impacto do Bolsa Família é baseado na comparação entre indivíduos que participam e os que não participam do programa social. Como a seleção dos beneficiados da política, no caso deste estudo, não ocorreu de forma aleatória, uma simples comparação entre os grupos (de tratamento e de controle) não seria correta. Isso porque o efeito do programa pode ser função de variável background (escolaridade do chefe de família, por exemplo), que, por sua vez, pode ser diferente entre os grupos. Assim, foram comparados indivíduos semelhantes entre as características observáveis, utilizando-se o matching ou pareamento das variáveis.

O PSM foi criado por Rosenbaum e Rubin (1983) para resolver o problema da multidimensionalidade do pareamento. Mostrou-se que o método pode ser implantado a partir de uma única variável de controle, o propensity score $P(X)$, definido como a probabilidade condicional de um indivíduo receber benefício, dadas as suas características observáveis (ver expressão 6) (ROSENBAUM; RUBIN, 1983):

$$
P(X)=\operatorname{Pr}(D=1 \mid X)
$$

A multidimensionalidade do pareamento pode ser solucionada por meio do propensity score, uma vez que a probabilidade se vincula a apenas um escalar. Na expressão $6, X_{i}$ (vetor das características observáveis) pode ser, então, substituído pelo escalar $P(X)$ :

$$
\begin{aligned}
& E\left(Y_{1}-Y_{0} \mid D=1, P(X)\right)=E\left(Y_{1} \mid D=1, P(X)\right)-E\left(Y_{0} \mid D=0, P(X)\right), \text { então } \\
& \left(Y_{0}, Y_{1} \perp D \mid P(X)\right)
\end{aligned}
$$

Com o propensity score, é possível ajustar todo viés existente entre os grupos de controle e de tratamento (ROSENBAUM; RUBIN, 1983). Entretanto, para a aplicação desse método, é preciso assumir duas hipóteses:

1) Balanceamento das características observáveis. A seleção da amostra requer que a participação no programa independa dos resultados e seja condicional nas covariáveis.

2) Existência de um suporte comum, isto é, não comparar o incomparável, o que geraria viés na avaliação. Então, $0<P(X)<1$, para cada grupo de controle existe um de tratamento correspondente. (HECKMAN et al, 1997). 


\subsection{Cálculo do Propensity Score}

Em geral o Propensity Score $P(X)$ não é conhecido, por isso é necessário estimá-lo. De posse de amostras dos grupos de controle e de tratamento, estima-se uma regressão logit para obter a probabilidade dos indivíduos participarem dos programas sociais, dadas as suas características observáveis $\left(X_{i}\right)$.

Nessa regressão, as variáveis independentes são aquelas que podem afetar a participação ou não do indivíduo na política ou programa de transferência social. A variável dependente é uma dummy que assume valor 1 para o caso do grupo de tratamento (chefes de família beneficiados) e zero para o grupo de controle.

Após a estimativa do propensity score, são obtidos subgrupos dentro do grupo de controle com probabilidades semelhantes às dos indivíduos do grupo de tratamento. Em seguida, para cada bloco do propensity score, testa-se se a média de cada variável preditora utilizada no modelo não difere entre tratamento e controle.

Se identificada distinção entre os grupos, deve-se especificar um modelo menos parcimonioso para a estimativa do propensity score. Caso contrário - se todos os testes para cada variável dentro de cada intervalo mostrarem que a média não difere significantemente -, um número de blocos ou estratos será definido, prosseguindo-se com o cálculo do Efeito Médio com Pareamento Estratificado (ATT).

\subsubsection{Cálculo do Efeito Médio do Programa com Pareamento Estratificado (ATT)}

Entre os diversos métodos de pareamento ou matching existentes na literatura, optou-se por utilizar o de pareamento estratificado (stratification matching). Formalmente, considera-se a distribuição de beneficiados e não beneficiados pela política pública em $n$ blocos ou estratos, de forma que a média das estimativas de propensity score para os dois grupos não apresente diferença estatisticamente significante em cada estrato. Se Y é a variável de interesse, o primeiro passo é calcular as diferenças de desempenho entre os beneficiados e não-beneficiados dentro dos estratos:

$$
\Delta_{e}^{S}=\frac{\sum_{i \varepsilon S(e)} Y_{i}^{B}}{N_{e}^{B}}-\frac{\sum_{i \varepsilon S(e)} Y_{i}^{N B}}{N_{e}^{N B}}
$$

em que $Y_{i}^{B}$ e $Y_{i}^{N B}$ correspondem aos resultados observados para os indivíduos dos grupos $i$ e $j$, respectivamente, dos grupos dos beneficiados e não-beneficiados (de controle). $\mathrm{O}$ resultados final é representado por:

$$
\Delta^{S}=\sum_{e=1}^{m} \Delta_{e}^{S} \frac{N_{e}^{B}}{N^{B}}
$$




\subsection{Amostra e Variáveis Utilizadas no Modelo}

Os dados utilizados neste estudo são provenientes de pesquisa de campo realizada pelo PADR-Ufrpe/Fadurpe com 838 famílias de agricultores familiares de 32 municípios dos estados da Paraíba (Cariri), Ceará (Sertão Central), Rio Grande do Norte (Apodi) e Sergipe (Sertão), no ano de 2005 (PROJETO DOM HELDER CÂMARA, 2006).

Observa-se que a renda média mensal das famílias que compõem a amostra é de $R \$ 240$ (Tabela 1). A idade média é de 44 anos. Já a escolaridade, que varia de zero a 16 anos de estudo, apresenta média bastante baixa, de três anos. Do total dos indivíduos amostrados, $63 \%$ são do sexo masculino, $29 \%$ são brancos, $31 \%$ são aposentados e $23 \%$ são beneficiários do Programa Bolsa Família (Tabela 2). O valor médio mensal do benefício recebido pelas famílias participantes é de $\mathrm{R} \$ 29,30$.

Tabela 1. Estatísticas básicas

\begin{tabular}{lcccc}
\hline & Renda & Idade & Anos de estudo & Consumo \\
\hline Média & 240,92 & 44 & 3 & 170,3 \\
Máximo & $3.063,33$ & 93 & 16 & 609,21 \\
Mínimo & 0 & 18 & 0 & 0 \\
\hline
\end{tabular}

Fonte: Dados pesquisa de campo (2005).

Tabela 2. Freqüência das variáveis dummies

\begin{tabular}{lcccccccc}
\hline & \multicolumn{2}{c}{ Gênero } & \multicolumn{2}{c}{ Raça } & Aposentadoria & \multicolumn{2}{c}{ Bolsa Família } \\
\hline & Masc. & Fem. & Branco & $\begin{array}{c}\text { Não } \\
\text { Branco }\end{array}$ & Sim & Não & $\begin{array}{c}\text { Benefi- } \\
\text { ciário }\end{array}$ & $\begin{array}{c}\text { Não } \\
\text { Benefi- } \\
\text { ciário }\end{array}$ \\
\hline Freqüência & 527 & 311 & 246 & 591 & 259 & 579 & 189 & 649 \\
Percentual & 0,63 & 0,37 & 0,29 & 0,71 & 0,31 & 0,69 & 0,23 & 0,77 \\
\hline
\end{tabular}

Fonte: Dados pesquisa de campo (2005).

O primeiro passo foi selecionar as variáveis independentes a serem incluídas no modelo logit - utilizado para calcular a probabilidade dos indivíduos participarem do programa de transferência social. Em seguida, esses indivíduos ou famílias foram agrupados ou pareados de acordo com a semelhança existente entre a probabilidade dos mesmos serem beneficiários do Programa Bolsa Família. A Tabela 3 apresenta as variáveis utilizadas no modelo logit.

Com a obtenção dos estratos a partir da estimativa do propensity score, é possível avaliar o impacto do recebimento do Bolsa Família sobre o consumo de alimentos das famílias. Para isso, a variável despesa com consumo de alimentos de cada indivíduo foi agregada para a unidade familiar. 
Tabela 3. Variáveis utilizadas no modelo logit

\begin{tabular}{lr}
\hline Variável & \multicolumn{1}{c}{ Descrição } \\
\hline $\begin{array}{l}\text { Renda Total } \\
\text { Idade }\end{array}$ & $\begin{array}{r}\text { Renda familiar anual, líquida do recebimento do Bolsa Família } \\
\text { Idade ao quadrado }\end{array}$ \\
$\begin{array}{l}\text { Criança } \\
\text { Área Construída }\end{array}$ & $\begin{array}{r}\text { Dummy que assume valor } 1 \text { caso a família tenha filhos de até 17 anos } \\
\text { Aposentado }\end{array}$ \\
$\begin{array}{l}\text { Escolaridade } \\
\text { Gênero }\end{array}$ & Dummy de aposentadoria \\
Raça Branca & Número de anos de estudo do chefe \\
Território Apodi & Dummy de raça do chefe da família que assume valor 1 para Branco \\
Território Sertão & Dummy para o território de Apodi \\
Central & Dummy para o território do Sertão Central \\
Território Cariri & Dummy para o território de Cariri \\
Banheiro & Dummy que assume valor 1 caso a família tenha banheiro na residência \\
Alvenaria & Dummy que assume valor 1 caso a residência seja construída com alvenaria \\
Canalização & Dummy que assume valor 1 para a residência que possua água encanada \\
\hline
\end{tabular}

Fonte: Elaboração própria.

\section{Resultados e Discussão}

Os resultados estão divididos em duas seções. Na seção 3.1, são feitas considerações sobre o modelo logit, construído para estimar o propensity score. $\mathrm{Na}$ seção 3.2, são analisados os resultados obtidos para as estimativas do efeito do tratamento.

\subsection{Estimação e Discussão do modelo logit}

A Tabela 4 apresenta o resultado para o modelo propensity score estimado. As especificações finais foram obtidas por meio de várias tentativas, a fim de se chegar a um modelo com maior número de variáveis. Isso porque quanto maior o número de variáveis incluídas, melhor é o pareamento entre os grupos de tratamento e de controle.

A análise dos preditores permite avaliar que, em geral, as variáveis apresentam os sinais esperados. Em um primeiro momento, observaram-se as variáveis relacionadas às características do chefe do domicílio, como gênero, raça, idade, idade $^{2}$ e se é aposentado. As variáveis gênero e raça não são estatisticamente significativas a menos de $10 \%$, de modo que não garantem aumento ou redução na probabilidade de recebimento do benefício pela família. 
A variável idade ao quadrado indica que, quanto maior a idade do chefe de família, maiores são as chances de recebimento do benefício. A partir de um determinado ponto, contudo, o cenário se inverte, diminuindo a probabilidade de inserção da família nos programas sociais. Ser aposentado também influi negativamente nas chances de recebimento.

Quanto à educação do chefe de família, a variável anos de estudo não se mostrou significativa, apesar do sinal negativo no coeficiente. Uma possível explicação pode estar associada à homogeneidade dos indivíduos que constituem a amostra em relação à escolaridade.

Variáveis relacionadas às condições de moradia da família, entre elas a existência de banheiro na residência e materiais usados na construção da casa, não são estatisticamente significantes a $10 \%$. De acordo com o sinal positivo do coeficiente da variável água encanada, é possível afirmar que a família que possui em sua residência água encanada tem maior chance de receber o benefício do programa social.

Variáveis que se relacionam com as características das famílias, como renda, presença de crianças e território, também apresentaram o sinal esperado. Com relação à renda, quanto maior o valor, menor é a probabilidade de participação no programa. $\mathrm{O}$ sinal positivo do coeficiente associado à variável criança por domicílio mostra que a residência composta por casais com filhos aumenta a chance de a família se enquadrar como beneficiária. Quanto ao território, quem mora no Sertão de Sergipe tem a maior probabilidade de receber o beneficio.

Tabela 4. Coeficientes estimados pelo modelo logit

\begin{tabular}{lcc}
\hline Variáveis & Coeficiente & Desvio Padrão \\
\hline Renda Total & $-4,7 \mathrm{E}-5^{* * *}$ & 0,000 \\
Idade $^{2}$ & $-9,9 \mathrm{E}-4^{* *}$ & 0,001 \\
Idade & $0,092^{* *}$ & 0,047 \\
Criança & $0,355^{*}$ & 0,053 \\
Área Construída & $-3,01 \mathrm{E}-3$ & 0,003 \\
Aposentado & $-0,167^{* * *}$ & 0,239 \\
Anos Estudo & $-0,016$ & 0,028 \\
Gênero & $-0,272$ & 0,192 \\
Raça Branca & $-0,079$ & 0,203 \\
Território Apodi & $-1,066^{*}$ & 0,276 \\
Território Sertão Central & $-0,696^{*}$ & 0,268 \\
Território Cariri & $-0,594^{* *}$ & 0,266 \\
Banheiro & $-0,361$ & 0,284 \\
Alvenaria & 0,402 & 0,328 \\
Canalização & $0,107^{* * *}$ & 0,200 \\
Intercepto & $-2,698^{*}$ & 1,042 \\
\hline
\end{tabular}

Nota: * Significante a 1\%, ** Significante a $5 \%,{ }^{* * *}$ Significante a $10 \%$.

Fonte: Elaboração própria - resultados gerados a partir dos dados da pesquisa de campo (2005). 
Após calcular a probabilidade de participação com o modelo logit, realizou-se o pareamento das famílias pertencentes aos grupos de controle e tratamento de acordo com a probabilidade que as mesmas têm de serem elegíveis como beneficiárias do programa social, dadas as suas características observáveis.

O resultado do pareamento, ou seja, a obtenção de subgrupos dentro do grupo de controle com valores de escores similares aos dos indivíduos do grupo de tratamento, pode ser observado na Tabela 5. Obteve-se quatro estratos de famílias com chances de receberem o Bolsa Família.

Tabela 5. Limite inferior, número de tratamentos e número de controles para cada bloco

\begin{tabular}{cccc}
\hline $\begin{array}{c}\text { Limite Inferior dos Estratos } \\
\text { do Propensity Score }\end{array}$ & Controle & Tratamento & Total \\
\hline 0 & 243 & 17 & 260 \\
0,125 & 269 & 62 & 331 \\
0,25 & 107 & 72 & 179 \\
0,5 & 30 & 38 & 68 \\
\hline Total & 649 & 189 & 838 \\
\hline
\end{tabular}

Fonte: Elaboração própria.

\subsection{Impacto do Programa Bolsa Família sobre os Gastos com Alimentos}

Após o pareamento estratificado, calcula-se o impacto do Programa Bolsa Família sobre as despesas de alimentos das famílias beneficiadas (ATT). O método de pareamento dividiu a variação dos escores de propensão em intervalos, de modo que, em cada um destes, as unidades tratadas e de controle apresentassem na média a mesma probabilidade de receber o benefício.

Em seguida, obteve-se o resultado quanto ao impacto da transferência de renda sobre o consumo alimentar. Em cada intervalo ou bloco, calculou-se a diferença dos resultados médios entre os grupos participantes e não-participantes. Ou seja, a média das despesas de consumo de cada bloco de famílias do grupo de tratamento foi comparada com a média de consumo do grupo de controle (Tabela 6).

Tabela 6. Cálculo do efeito do tratamento para as despesas com consumo de alimentos

\begin{tabular}{ccccc}
\hline Tratamento & Controle & ATT & Desv. Pad. & T \\
\hline 186 & 636 & 246,311 & 102,742 & 2,397 \\
\hline
\end{tabular}

Fonte: Elaboração própria. 
Na Tabela 6, nota-se que existe uma diferença positiva e significativa das despesas com alimentos entre os grupos de tratamento e de controle. $\mathrm{O}$ valor médio das despesas anuais para a família beneficiária é $\mathrm{R} \$ 246$ superior à média dos gastos totais das famílias não-participantes, cuja renda per capita mensal é de R\$ 100. Considerando que a média anual recebida por essas famílias é de R\$278, pode-se inferir que $88 \%$ desse valor é utilizado para consumo de alimentos. Portanto, o programa de transferência Bolsa Família exerce um impacto positivo sobre o consumo de alimentos das famílias selecionadas.

Esse resultado está de acordo com os obtidos nas análises do Ministério da Saúde (2004, 2005), indicando que o Programa Bolsa Família apresenta dados semelhantes aos estimados para o Programa Bolsa Alimentação, que o antecedeu. Ressalte-se que o Programa Bolsa Família amplia os beneficiários, em função da renda, atingindo as famílias rurais de baixa renda e não apenas aquelas consideradas prioritárias de um ponto de vista nutricional (gestantes, nutrizes e crianças com menos de cinco anos). Essa maior abrangência constitui uma ampliação no sentido de beneficiar a pobreza rural como um todo. O fato de essa ampliação não ter afetado o gasto com alimentos é indicativo de que a condicionalidade deve estar ligada ao modo como a renda transferida é percebida pela família e ao processo de decisão quanto a seu uso. Destaca-se o papel da mulher como gestora dos recursos recebidos do Bolsa Família.

\section{Conclusões}

Programas de transferência condicionada de renda são políticas sociais correntemente empregadas para combater e reduzir a pobreza em diversos países. No curto prazo, esses programas visam aliviar os problemas decorrentes da situação de pobreza, sendo que, no longo prazo, o objetivo é investir no capital humano, quebrando o ciclo intergeracional da pobreza.

Uma questão que surgiu a respeito dessa política é como verificar a eficiência do Bolsa Família sobre o bem-estar das famílias. Muitos trabalhos avaliam os efeitos diretos do programa, como o aumento do número de matrículas e a freqüência escolar das crianças beneficiadas. Entretanto, este estudo se propôs a estudar os impactos indiretos da política de transferência de renda condicional, como o aumento do consumo de alimentos pelas famílias selecionadas pelo programa.

Dada a restrição imposta pelos dados, optou-se pelo uso do método propensity score, o qual permite a utilização de informações coletadas em um ponto do tempo. A situação ideal seria possuir dados que permitissem a análise da mesma família em dois períodos do tempo, o que produziria resultados mais robustos. Entretanto, o pareamento dos grupos a partir do propensity score, segundo a literatura, é bastante adequado para a redução do viés de seleção. 
Os resultados estimados por meio do propensity score indicaram que existem quatro grupos de pareamento, ou seja, quatro subgrupos de famílias, dentro dos grupos de controle e de tratamento, com probabilidades semelhantes de serem elegíveis como beneficiárias do Bolsa Família.

O resultado estimado ao se calcular a diferença de média do consumo entre os subgrupos das famílias participantes e não-participantes sugere que existe uma diferença significativa e positiva entre média de consumo de alimentos dos grupos de tratamento e de controle. $\mathrm{O}$ valor médio dos gastos mensais das famílias beneficiárias é R $\$ 246$ superior à média dos gastos totais das famílias não-participantes. Considerando que a média anual recebida por essas famílias é de $\mathrm{R} \$ 278$, pode-se inferir que $88 \%$ desse valor é utilizado para consumo de alimentos. A elasticidade-renda por alimento da renda de transferência é bem maior que a elasticidade referente à renda total.

Portanto, existe um efeito positivo das transferências monetárias sobre o consumo alimentar das famílias beneficiárias. Esse resultado vai de acordo com os obtidos pelo Ministério da Saúde $(2004,2005)$, mostrando que o Programa Bolsa Família apresenta resultados semelhantes aos estimados para o Programa Bolsa Alimentação, que o antecedeu.

\section{Referências Bibliográficas}

ATTANAZIO, O., BATTISTIN, E., FITZSIMONS, E., MESNARD, A., VERA-HERNÁNDEZ, M. (2005). How effective are conditional cash transfers? Evidence from Colombia. London: The Institute for Fiscal Studies. Disponível em http://www.ifs.org.uk/publications.php.

BECKER, S.O., ICHINO, A. (2002). Estimation of average treatment effects base do propensity score. Stata Journal, v.2, n.4, p.358-357.

BOURGUIGNON, F., FERREIRA, F., LEITE, P. (2003). Conditional Cash Transfers, Schooling and Child Labor: Simulating Bolsa Escola. Working Paper 2003-07, Delta, University of Paris.

CAMARGO, J. M., FERREIRA, F.H.G. (2001). O benefício social único: Uma proposta para reforma da política social no Brasil. Texto para discussão, departamento de economia, PUC-RIO.

CARDOSO, E., SOUZA, A. P. (2004). The Impact of Cash Transfer on Child Labor and Scholl attendance in Brazil. n.04, w. 07. Disponível em: http://www.vanderbilt.edu/econ.

DEHEJIA, R. (2005). Practical propensity score matching: a reply to Smith and Todd. Journal of Econometrics, v.125, n.1-2, p. 355-364. 
FERNANDES, R., FELÍCIO, F. (2003). Impacto dos programas oficiais de transferência de renda sobre a pobreza nas unidades da federação brasileira. Brasília.

FERRO, A. R., KASSOUF, A. L. (2003). Avaliação dos impactos dos Programas Bolsa Escola sobre o Trabalho Infantil no Brasil. Disponível em http://www.cepea.esalq.usp.br. Banco Mundial, Rural Poverty Alleviation in Brazil.

HECKMAN, J., ICHIMURA, H., TODD, P. (1997). Matching as an econometric evaluation estimator: evidence from evaluating a job training program. Review of Economic Studies, v. 64(4), n. 221, p.605-654.

HOLZMANN, R., JORGENSEN, O. (2000). Manejo social del riesgo: un nuevo marco conceptual para la protección social y más allá, documento de trabajo, № 0006, Washington, D.C., Banco Mundial. Disponível em http://www1.worldbank.org.sp.safetynets/.

LAVINAS, L. (1998). Programas de garantia de renda minima: perspectivas brasileiras.Textos para discussão - Ipea, No. 596.

MULLER, C. E BIBI, S. (2006). Focused Targeting against Poverty Evidence from Tunisia. IDEP Working Papers 0602, Institut d'economie publique (IDEP), Marselha, França. Disponível em http://www.vcharite.univ-mrs.fr/idep/document/dt/dt0602.pdf.

PROJETO DOM HELDER CAMARA (2006). Relatorios de Perfil de Entrada dos Territorios do Sertao Central do Ceara, do Apodi, do Cariri Paraibano e do Sertão Sergipano, Recife, PADR-UFRPE/FADURPE.

RAVALLION, M. (2003). Targeted Transfers in Poor Countries: Revisiting the Trade-offs and Policy Options, Social Protection Discussion Paper, № 0314, Washington, D.C., Banco Mundial. Disponível em http://www1.worldbankorg/sp/safetynets/.

ROSEMBAUM, P. R., RUBIN, D. B. (1983). The central role of propensity score in observational studies for causal effects. Biometrika, v.70, n.1, p. 41-55.

SKOUFIAS, E., PARKER, S. (2001). Conditional Cash Transfers and Their Impacts on Child Work and Schooling: Evidence from the Progresa Programa in Mexico, FCND Discussion Paper, № 123, Washington, D.C., Instituto Internacional de Investigación de las Políticas Alimentarias (IFPRI). Disponível em http://www.ifpri.org.br.

VILLATORO, P. (2005). Programas de transferências monetárias condicionadas: experiência en América Latina. Revista de la Cepal . 